\title{
Topologically Induced Confinement of Collective Modes in Polycrystalline Graphene Nano-Cones: Measured by Momentum Transfer Dependent STEM-VEELS
}

\author{
F.S. Hage ${ }^{1,2}$, Q.M. Ramasse ${ }^{3}$, D.M. Kepaptsoglou ${ }^{3}$, Ø. Prytz $^{2}$, G. Helgesen ${ }^{1,2}$, and R. Brydson ${ }^{4}$ \\ 1. Physics Department, Institute for Energy Technology, Kjeller, NO- 2027, Norway. \\ 2. Department of Physics, University of Oslo, Oslo, NO-0316, Norway. \\ 3. SuperSTEM Laboratory, SciTech Daresbury, Daresbury, WA4 4AD, U.K \\ 4. Institute for Materials Research, SPEME, University of Leeds, Leeds, LS2 9JT, U.K
}

In the rapidly expanding field of plasmonics, localization of electromagnetic energy is a key property, the accurate control of which allows for nanoscale manipulation of optical properties. Present and future applications of plasmonic devices include highly efficient photovoltaics, ultrafast computing, molecular tweezers, optical microscopy below the diffraction limit, and even cloaking devices [1]. In this study, the effect of topological defects on the confinement of collective modes in multiwalled carbon nano-structures is investigated at high spatial resolution by momentum transfer dependent valence electron energy loss spectroscopy (VEELS) in the aberration corrected dedicated scanning transmission electron microscope (STEM). The degree of plasmon localization is determined from the momentum transfer dependence (i.e. the dispersion) of the $\pi$ and $\pi+\sigma$ volume plasmon excitations, where a vanishingly small dispersion corresponds to a localized state.

Polycrystalline graphene nano-cones exhibit easily distinguishable macroscopic apex angles $\left(0^{\circ}\right.$, $112.9^{\circ}, 84.6^{\circ}, 60^{\circ}, 38.9^{\circ}$ and $19.2^{\circ}$ ), the value of which is attributed to a distinct number of pentagonal apical defects $(P=0-5)$, where $P=0$ corresponds to the case of a flat disc [2]. Thus nano-cones and discs allow for direct correlation of the number of pentagons with the degree of plasmon confinement. When five pentagons are present at the cone apex (see example in Fig. 1(a)), the $\pi$ and $\pi+\sigma$ volume plasmons both show a vanishingly small dispersion (Fig. 1(c)). This indicates a high degree of confinement, in stark contrast to the graphite-like delocalization [3] indicated by the approximately parabolic dispersion (Fig. 1(c)) of a disc (see example in Fig. 1(b)). Possible implications of the measured slightly negative cone $(P=5) \pi$ and $\pi+\sigma$ plasmon dispersions will also be discussed. All data shown here were acquired with a Nion UltraSTEM100 operated at $60 \mathrm{kV}$ in order to minimize irradiation damage [4].

References

[1] J. A. Dionne and H. A. Atwater, MRS Bulletin, 37 (2012)717

[2] A. Krishnan et al., Nature, 388 (1997) 451

[3] H. Venghaus, Physica Status Solidi (b), 66 (1974) 145

[4] This work was supported by the Research Council of Norway under Contract No. $191621 / \mathrm{V} 30$ 

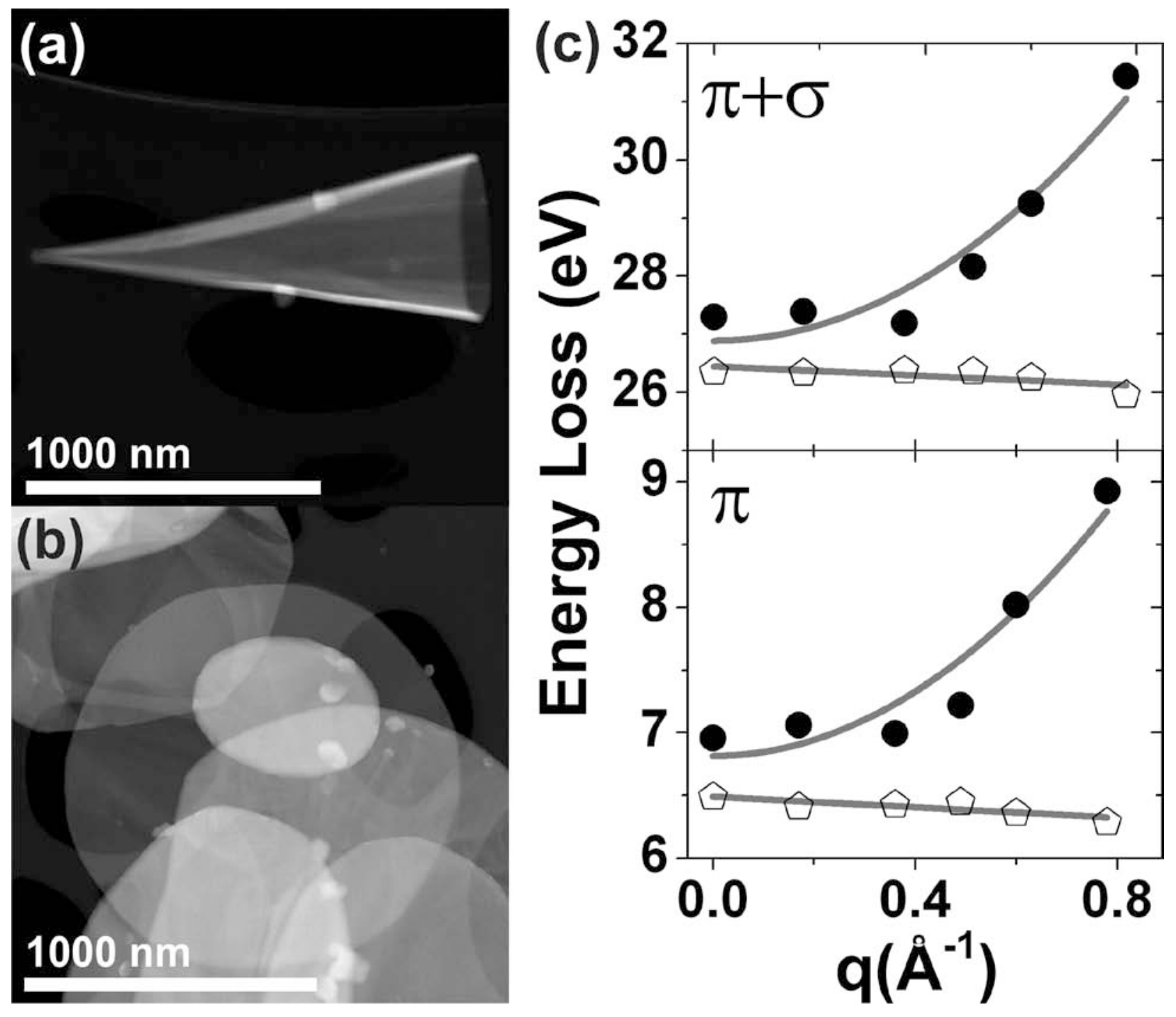

Figure. 1. (a) HAADF image of a nano-cone with five pentagons at the apex. (b) HAADF image of overlapping nano-discs of varying sizes. (c) The $\pi$ and $\pi+\sigma$ volume plasmon dispersions from a disc $(\bigcirc)$ and the tip of a cone $(P=5)(\triangle)$. 